\title{
Finding the Best QoS Path in a Gilbert Channel Network
}

\author{
Edwin Soedarmadji \\ California Institute of Technology, Pasadena, CA 91125, USA, \\ edwin@systems.caltech.edu
}

\begin{abstract}
Many different types of modern wired and wireless communication links can be mathematically described as discretetime Gilbert channels. In this extended abstract, we present an exact method of calculating the best path in a network of discretetime Gilbert channels, each of which is defined as a Markov chain with two states. In the "Good" state of the chain, the channel produces no erasure, and in the "Bad" state of the chain, the channel produces an erasure. Our method relies on a modified version of the Dijkstra's algorithm, which we customize to operate on sets of Gilbert channel parameters, instead of real numbers. We prove that the Gilbert channels obeys a certain set of algebraic properties which makes it compatible with our algorithm.
\end{abstract}

\section{INTRODUCTION}

To the first approximation, many different types of modern wired and wireless communication links can be considered memoriless. Thus, depending on the types of signal used, these links can be mathematically modeled as binary erasure channels, binary symmetric channels, or AWGN channels, etc. However, in many applications, accurate behavior and performance analysis has to take into account the fact that in reality, channel distortion exhibits memory.

The Gilbert channel model [1] is one of the simplest models for channels with memory. Mathematically, it is nothing more than a Markov chain with two states: $G$ and $B$, with their outgoing transition probability values denoted by $p$ and $q$, respectively. In this extended abstract, we assign $B$ to be the state where undesirable events occur, e.g., packet loss, packet error, fading higher than a predetermined threshold, etc.

Despite (also because of) its apparent simplicity, the Gilbert Channel Model (GCM) has been widely used in analyzing a wide variety of networks [2]. For example, GCM is used to analyze the performance of slotted ALOHA over fading communications channels [3], or correlated loss over TCP/IP networks [4]. Other relevant examples include performance analysis of real-time wireless communications [5]. For many wireless fading channels, GCM is a very attractive alternative to sophisticated models such as Hidden Markov models (HMMs) [6]. For one, GCMs are analytically tractable. In addition, it can easily produce packet-level network QoS.

GCM becomes even more important with the advent of Universal Mobile Telecommunications Systems (UMTS) networks where multimedia (especially speech) and data packets will have to coexist in the underlying common Wideband CodeDivision Multiple Access (WCDMA) networks.
The recent UMTS recommendations for QoS measures is based on a set of user satisfaction assessments of individual speech and data sessions [7]. For speech services, user satisfaction drops significantly in the presence of long spans (in terms of packets) of service outage. For data services, disruption and termination comes from successive retransmissions.

These new QoS measures require the next level of approximation of behavioral analysis that is not available from system outage probability analysis (which inherently assumes zero correlation between the outages) commonly found in CDMA literature [8], [9]. Recently, detailed analysis (in the context of QoS and capacity) of WCMDA [10], [11] incorporates correlated outage behavior by using GCM.

This extended abstract attempts to outline our method of finding the best QoS path in networks where GCM can be applied, and wherever shortest path routing is used. In particular, it also applies to the WCDMA case that we just discussed.

\section{Gilbert Channel Network}

As previously mentioned, a discrete-time Gilbert Channel (GC) is defined as a Markov chain with two states, $G$ and $B$, whose outgoing transition probability values are denoted by $p$ and $q$, respectively, with $0 \leq p, q \leq 1$.

The Markov chain produces a deterministic binary output value that indicates whether or not the channel produces an erasure event. In the $G$ (or "Good") state, the output is a 0 , which indicates no erasure, whereas in the $B$ (or "Bad") state, the output is a 1 , which indicates a channel erasure. Mathematically, we can represent the space of all GC as the space $\Lambda=[0,1] \times[0,1]$, and a particular GC instance by a point $\lambda=(p, q) \in \Lambda$. In particular, when $p=0$ or $q=0$, the GC converges to a deterministic steady-state behavior.

Suppose we represent all networks by the standard $\mathcal{G}=$ $(V, E)$ node- and edge-sets. Then a Gilbert Channel Network $(\mathrm{GCN})$ is just $\mathcal{G}$ with the additional specification that its edge weights are taken from $\Lambda$. This brings out several important questions about GCNs. First, is it possible to combine GCN edges into a path, and if so, is it possible to derive a combined path weight from its edge weights? Second, is it possible to compare path weights to find the best path in a GCN? Third, can we cast this problem as a generalized shortest path problem that can be solved using the Generalized Dijkstra's Algorithm (GDA) [12]? If so, we say that the GCN is compatible with the GDA, and the best path can be found in $O\left(V^{2}\right)$. 
As always, we obtain the most definitive answer by paraphrasing the questions into its equivalent formal mathematical questions. If we define an algebra $\mathbf{B}$ by combining $\Lambda$ and two operators: $\oplus$ and $\preceq$ to represent the general operations of (1) adding two edge weights together (to ultimately construct a path), and (2) comparing two weight values, the questions is identical to proving the following proposition:

Proposition 1. An algebra $\mathbf{B}=(\Lambda, \oplus)$ and a total order $\preceq$ is compatible with the GDA if and only if it satisfies all the properties in the set denoted by $\mathbf{P}$ below:

P1 is a commutative monoid, that is, for $a, b, c \in \Lambda$ :

- $\Lambda$ is closed under $\oplus: a \oplus b \in \Lambda$;

- $\oplus$ is associative : $a \oplus(b \oplus c)=(a \oplus b) \oplus c$;

- 0 is the identity : $a \oplus 0=0 \oplus a=a$;

- $\oplus$ is commutative : $a \oplus b=b \oplus a$.

P2 There exists $\infty \in \Lambda \mid a \oplus \infty=\infty \oplus a=\infty$.

$\mathbf{P 3} \preceq$ is a total order on $\Lambda$, i.e., $\preceq$ is :

- reflexive: $a \preceq a$;

- anti-symmetric: if $a \preceq b$ and $b \preceq a$ then $a=b$;

- transitive: if $a \preceq b$ and $b \preceq c$ then $a \preceq c$;

- total: for every $a, b \in \Lambda$ either $a \preceq b$ or $b \preceq a$.

P4 There exists the least element 0 that satisfies $0 \preceq a$.

P5 $a \oplus c \prec b \oplus c$ if $a \prec b$ and $c \in \Lambda-\{\infty\}$.

The Generalized Dijkstra's Algorithm (GDA) below [12] is a modified version of Dijkstra's Algorithm that can operate on any metric of choice as long as the metric is compatible. In this extended abstract we attempt to provide a sketch the proofs that B is compatible with the GDA, deferring the complete proof to the full paper version of this abstract.

In the following listing, $\mathcal{G}$ is the $\mathrm{GCN}$, while $s$ is the source node of $\mathcal{G}$. The functions $\beta$ and $N$ return (1) the edge weight connecting its two argument nodes, and (2) the neighbors of its argument, respectively. The arrays $l$ and $\pi$ keep track of cumulative path length and predecessor node, respectively.

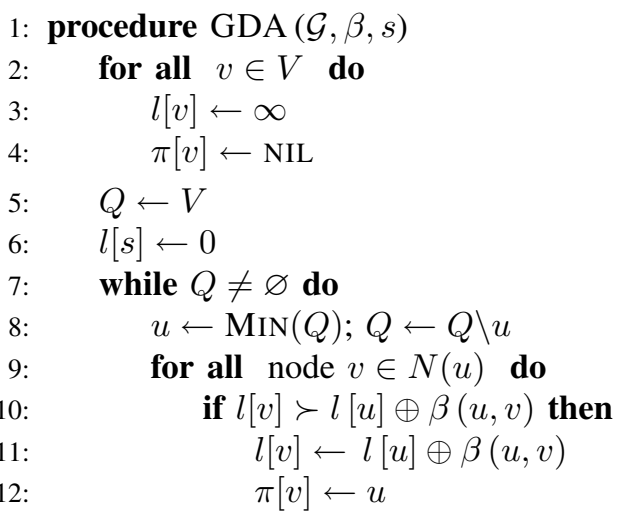

Interested readers can consult [13] for a description of the standard Dijkstra's Algorithm, which is practically identical to the GDA except for the relaxation step, where the $\oplus$ and $\preceq$ operators act on a generalized metric space $\Lambda$ (instead of the equivalent step in DA, where + and $\leq$ operators act on $\mathbb{R}$ ). We shall shortly define $\preceq$ for our channel.
In our previous work, we presented methods based on the GDA to find the best QoS path in networks containing (exclusively of) Binary Erasure Channels (BEC) [14], Binary Symmetric Channels (BSC) [15], and with a very high probability, the Additive White Gaussian Noise (AWGN) channels.

Since the GC is a generalization of the BEC (a BEC with loss parameter $p$ is just a $\mathrm{GC}$ with $q=1-p$ ), the results presented here extend our previous results for the BEC network by introducing a simple Markovian memory.

Finally, before we begin presenting the sketch of the proof, first we have to precisely define in the next section what the operators $\oplus$ and $\preceq$ really mean, and define more precisely the various symbols for our network problem.

\section{Network Model}

The Gilbert Channel Network (GCN) is modeled as a digraph $G=(V, E)$, where $V, E$, and $\Pi$ are the node, edge and path sets of $G$. Two nodes $s$ and $d \in V$ are the source and destination nodes, and $\Pi \subset \Pi$ denotes the set of all paths from $s$ to $d$.

What makes the GCN unique is the space of its edge weight values $\boldsymbol{\lambda} \in \Lambda$ that contains all pairs of parameter values $(p, q)$, whose components represent the transition probability $p$ from the desirable state $G$ to the undesirable state $B$, and vice versa for $q$. In this definition, the probability values of staying in the same states are $1-p$ and $1-q$, respectively. Each edge $e_{i} \in E$ has its own edge weight $\boldsymbol{\lambda}_{i} \in \Lambda$. The special value $\boldsymbol{\lambda}=\infty$ denotes the absence of connection between two nodes.

A path $\pi \in \Pi$ whose nodes $V_{\pi} \subset V$ are connected by $E_{\pi} \subset E$ is denoted by either $\left\langle v_{0}, \ldots, v_{J}\right\rangle$ or $\left\langle e_{1}, \ldots, e_{J}\right\rangle$. The symbol $\left\langle v_{i}, v_{i+1}\right\rangle$ denotes the edge (path) connecting the two (non-) adjacent nodes $v_{i}$ and $v_{i+1}$ in a network of GC's.

Define the function $X: \Lambda \rightarrow \mathcal{M}$ that measures a userdefined degree of reliability associated with a path or an edge. For convenience, let us define the function $\beta: \Pi \rightarrow \Lambda$ that maps a path $\pi$ (or an edge $e_{i}$ ) into a parameter $\boldsymbol{\lambda}_{\pi}$ (or $\boldsymbol{\lambda}_{i}$ ), and a shorthand notation $X(e)=X(\beta(e))$.

Let us assume that the addition operation is defined in $\Lambda$ and $\mathcal{M}$ and is denoted by $\oplus$. If $x_{1}=X\left(e_{1}\right), x_{2}=X\left(e_{2}\right)$, $\boldsymbol{\lambda}_{1}=\beta\left(e_{1}\right), \boldsymbol{\lambda}_{2}=\beta\left(e_{2}\right)$, and $\pi=\left\langle e_{1}, e_{2}\right\rangle$, then we say $x_{\pi}=x_{1} \oplus x_{2}$ and $\boldsymbol{\lambda}_{\pi}=\boldsymbol{\lambda}_{1} \oplus \boldsymbol{\lambda}_{2}$. For these expressions to make sense, the $\oplus, \Lambda$ and $\mathcal{M}$ have to obey the algebraic properties $\mathbf{P}$ that we discussed in the previous section. Having defined $\oplus$, we can now compute $x_{\pi}$ and $\boldsymbol{\lambda}_{\pi}$ in terms of $x_{i}$ and $\boldsymbol{\lambda}_{i}$ using a generalized summation: $x_{\pi}=\bigoplus x_{i}$ and $\boldsymbol{\lambda}_{\pi}=\bigoplus \boldsymbol{\lambda}_{i}$.

We call the pairs $(\Lambda, \oplus)$ and $(\mathcal{M}, \oplus)$ the $\mathbf{B}$ and $\mathbf{X}$ algebras, with their associated $\beta$ and $X$ functions, respectively. To calculate the optimal network path $\pi^{*}$, however, we need more than $\oplus, \beta$ and $X$ to evaluate expressions like these:

$$
\begin{aligned}
x^{*} & =\min _{\pi}\left\{x_{\pi} \mid \pi \in \Pi\right\} \\
\boldsymbol{\lambda}^{*} & =\min _{\pi}\left\{\boldsymbol{\lambda}_{\pi} \mid \pi \in \Pi\right\}
\end{aligned}
$$

We need a total order operator $\preceq$ acting on $\Lambda$ and $\mathcal{M}$ to evaluate expressions such as $x_{\pi} \preceq x_{\pi^{\prime}}$ and $\boldsymbol{\lambda}_{\pi} \preceq \boldsymbol{\lambda}_{\pi^{\prime}}$. Once the operator $\preceq$ is defined, then the above expression makes sense. 
For simplicity, the metrics induced from the coupling of $\preceq$ with $\mathbf{B}$ and $\mathbf{X}$ are also refered to as the $\mathbf{B}$ and $\mathbf{X}$ metrics, respectively. With reference to the source node $s$ and the destination node $d$, the optimal path $\pi^{*}$ is the path with the "shortest" length when measured in the $\mathbf{B}$ and $\mathbf{X}$ metrics, both of which use the $\preceq$ operator that is defined as follows:

Definition 2. $\boldsymbol{\lambda}_{1} \preceq \boldsymbol{\lambda}_{2}$ iff $X\left(\boldsymbol{\lambda}_{1}\right) \preceq X\left(\boldsymbol{\lambda}_{2}\right)$.

Before defining the $\oplus$ operator for $\mathbf{B}$ and $\mathbf{X}$, let us define the $X(e)$ function in this paper as the function that measures the long term probability of channel erasures of an edge $e$. This definition of $X(e)$ is compatible to the analysis in our previous work [14] of the standard memoriless erasure channel, which is a special case of the GC with $q=1-p$.

Denote by $S_{i}(t)$ the state at a particular (discrete) time $t$ of the Markov chain associated with the GC on an edge $e_{i}$, and by $F_{i}(t)$ the corresponding Markov chain output. For brevity, the dependence on $t$ is omitted from the notation when it is obvious from the context. For the memoriless case, the probability for observing the failure indicator $F_{i}$ is given by:

$$
P\left(F_{i}=f_{i}\right)= \begin{cases}1-p & , f_{i}=0 \text { (no failure) } \\ p & , f_{i}=1 \text { (failure). }\end{cases}
$$

The $\mathbf{B}$ algebra is such that any two adjacent edges $e_{1}$ and $e_{2}$ with parameters $\boldsymbol{\lambda}_{1}$ and $\boldsymbol{\lambda}_{2}$ (and their corresponding long term erasure probabilities $x_{1}$ and $x_{2}$ ) can be viewed as a single edge with parameter $\boldsymbol{\lambda}=\boldsymbol{\lambda}_{1} \oplus \boldsymbol{\lambda}_{2}=p_{1} \oplus p_{2}$ defined by:

$$
\begin{aligned}
& p_{1} \oplus p_{2}=1-\left(1-p_{1}\right)\left(1-p_{2}\right) \\
& x_{1} \oplus x_{2}=p_{1} \oplus p_{2}
\end{aligned}
$$

In the memoriless case, the scalar parameter that plays the role of $\boldsymbol{\lambda}$ is $p$, and from the law or large numbers, we know that the value of $X(\boldsymbol{\lambda})$ is identical to the value of probability function $P$ above. Therefore, in this case, the $\preceq$ operator is defined by the following relation: $\boldsymbol{\lambda}_{1} \preceq \boldsymbol{\lambda}_{2}$ iff $p_{1} \preceq p_{2}$.

To model channel erasures with memory, we return to our assumption that at each time $t$, the state $S_{i}(t)$ of an edge $e_{i}$ could either be Good (labeled $G$ ) or Bad (labeled $B$ ), and that $S_{i}(t)$ is Markovian. In the good state, the output $F_{i}(t)=0$ (no erasure), and in the bad state, the output $F_{i}(t)=1$ (erasure).

As is true with any two-state Markov Chain, a GC is completely defined by its transition probabilities. Let us denote the transition probability from the good to the bad state by $p=P\left(S_{i}(t)=B \mid S_{i}(t-1)=G\right)$, and from the bad to the good state by $q=P\left(S_{i}(t)=G \mid S_{i}(t-1)=B\right)$.

In a GCN, each edge $e_{i}$ is given an edge weight $\boldsymbol{\lambda}_{i}$ that is completely characterized by the vector $\left(p_{i}, q_{i}\right)$. As before, the $\mathbf{B}$ algebra is defined in such a way that any two adjacent edges $e_{1}$ and $e_{2}$ with parameters $\boldsymbol{\lambda}_{1}$ and $\boldsymbol{\lambda}_{2}$ can be viewed as a single edge with parameter $\boldsymbol{\lambda}=\boldsymbol{\lambda}_{1} \oplus \boldsymbol{\lambda}_{2}=p_{1} \oplus p_{2}$.

As expected, the definition of the $\oplus$ operator for the GCs is a little bit more complex than the definition of the $\oplus$ operator for the memoriless case. To define the $\oplus$ for GCs, we have to take an indirect approach that uses the definition of the $X$ function and its associated $\mathbf{X}$ algebra.
Like in the case of memoriless erasure channel, the function $X$ measures the long term erasure probability. The difference is that, for erasure channels with memory, Markov chains are involved and the function $X$ actually measures the stationary probability of finding the edge $e_{i}$ in the bad state, i.e.: $X_{B}\left(e_{i}\right)=P\left(S_{i}=B\right)$ (when the edge in question is not ambiguous, we simply refer to this quantity as $X_{B}$ ). This probability is given by the following expression:

$$
X_{s}\left(e_{i}\right)=P\left(S_{i}=s\right)= \begin{cases}\frac{q_{i}}{p_{i}+q_{i}} & , s=G \\ \frac{p_{i}}{p_{i}+q_{i}} & , s=B .\end{cases}
$$

As expected, $X_{G}+X_{B}=1$, which reflects the fact that for any observation, the edge $e_{i}$ has to be in one of the states.

Continuing our effort to define $\oplus$, we then observe that since the failure events for two adjacent edges $e_{1}$ and $e_{2}$ are independent of one another, the stationary probability of finding $e=\left\langle e_{1}, e_{2}\right\rangle$ in the good state is simply the product of $X_{G}\left(e_{1}\right)$ and $X_{G}\left(e_{2}\right)$. Switching to the $\mathbf{X}$ algebra, we claim that:

$$
\begin{aligned}
X_{G}(e) & =X_{G}\left(e_{1}\right) \oplus X_{G}\left(e_{2}\right) \\
& =X_{G}\left(e_{1}\right) X_{G}\left(e_{2}\right) \\
& =\frac{q_{1}}{p_{1}+q_{1}} \frac{q_{2}}{p_{2}+q_{2}} \\
& =\frac{q_{1} q_{2}}{\left(p_{1}+q_{1}\right)\left(p_{2}+q_{2}\right)} \\
& =\frac{q}{p+q}
\end{aligned}
$$

Equation (5) gives one equation for solving the two unknowns $p$ and $q$, which are the transition probabilities of the combined edge $e$. The other equation is from the definition of $p$ :

$$
p=1-\left(1-p_{1}\right)\left(1-p_{2}\right)
$$

The above equation mathematically states that for the edge $e$ to be in the Good state, both $e_{1}$ and $e_{2}$ have to be in their respective Good states. If either one of them makes a transition to the Bad state, then $e$ will also transition to its Bad state.

Lemma 3. Let $\boldsymbol{\lambda}_{1}=\left(p_{1}, q_{1}\right)$ and $\boldsymbol{\lambda}_{2}=\left(p_{2}, q_{2}\right)$. Suppose $\boldsymbol{\lambda}=(p, q)=\boldsymbol{\lambda}_{1} \oplus \boldsymbol{\lambda}_{2}$. If we denote by $p=p\left(p_{1}, q_{1}, p_{2}, q_{2}\right)$ and $q=q\left(p_{1}, q_{1}, p_{2}, q_{2}\right)$, then:

$$
\begin{aligned}
& p=1-\left(1-p_{1}\right)\left(1-p_{2}\right) \\
& q=p \cdot \frac{q_{1} q_{2}}{\left(p_{1}+q_{1}\right)\left(p_{2}+q_{2}\right)-q_{1} q_{2}}
\end{aligned}
$$

PROOF: We have already discussed the first equation and will not repeat the proof. The proof for $q$ is as follows. From the last equation in (5), we first solve for $q$,

$$
\begin{aligned}
q & =p \cdot \frac{X_{G}(e)}{1-X_{G}(e)} \\
X_{G}(e) & =\frac{q_{1} q_{2}}{\left(p_{1}+q_{1}\right)\left(p_{2}+q_{2}\right)}
\end{aligned}
$$

and substitute the full expression of $X_{G}(e)$. Following some algebraic simplification, $q$ can be easily obtained. 
Finally, to complete our discussion on the algebraic operators for GCs, we provide the following definition for $\preceq$. If we let $\boldsymbol{\lambda}_{1}=\beta\left(e_{1}\right)$ and $\boldsymbol{\lambda}_{2}=\beta\left(e_{2}\right)$, then:

$$
\boldsymbol{\lambda}_{1} \preceq \boldsymbol{\lambda}_{2} \quad \text { iff } \quad X_{B}\left(\boldsymbol{\lambda}_{1}\right) \preceq X_{B}\left(\boldsymbol{\lambda}_{2}\right) .
$$

Having defined $\oplus$ and $\preceq$, we are now ready to show that GCs have $\mathbf{B}$ (and $\mathbf{X}$ ) algebras that are compatible to the Generalized Dijkstra's Algorithm (GDA), a modified version of the original Dijkstra's Algorithm (DA), which has been proven to have a time-complexity of at most $O\left(V^{2}\right)$.

Theorem 4. The algebra $\mathbf{B}=(\Lambda, \oplus)$ in the memoriless case and its respective total order $\preceq$ satisfy all the properties in $\mathbf{P}$, and thus compatible with the GDA.

PROOF: We will prove the properties one by one. Recall that $\Lambda=[0,1]$. Let the variables $a, b$, and $c$ in $\mathbf{P}$ correspond to the parameters $p_{1}, p_{2}$, and $p_{3}$ (as defined in equation (3) of three consecutive edges $e_{1}, e_{2}$, and $e_{3}$.

P1 Except for closure, the monoid properties can be proven by performing an algebraic manipulation of equation (3). Recall that $\Lambda=[0,1] \cap \infty$. Closure is obvious if $a, b \in$ $[0,1]$. In the proof for P2, we idenfity the element $\infty \in$ $[0,1]$. Thus, closure is guaranteed when $a=\infty$ or $b=\infty$. This gives us one interpretation of $\infty$; i.e., it is an alias for an element in $[0,1]$. We can also adapt an alternative interpretation of an edge weight of $\infty$, using it as a notation to indicate the absence of any connection between two nodes. In this interpretation, $a \oplus b=\infty$, because if either $a=\beta\left(\left\langle v_{1}, v_{2}\right\rangle\right)=\infty$ or $b=\beta\left(\left\langle v_{2}, v_{3}\right\rangle\right)=\infty$, then $\beta\left(\left\langle v_{1}, v_{3}\right\rangle\right)=\infty$.

P2 The proof is obvious if $\infty$ is a notation for the absence of connection (as we just discussed), or alternatively, $\infty=$ $1 \in[0,1]$ also satisfies this property.

P3 The proof follows the properties of $\Lambda$, which is just $\mathbb{R}$.

P4 Same as above.

P5 The proof is obvious if $b=\infty$. However, if $b \neq \infty$, then by substituting $a, b$, and $c$ into (3) we obtain the two values $a \oplus c$ and $b \oplus c \in[0,1]$ given by:

$$
\begin{aligned}
& a \oplus c=1-(1-a)(1-c) \\
& b \oplus c=1-(1-b)(1-c) .
\end{aligned}
$$

Since both are in $[0,1]$, the order $\prec$ is just $<$, and the expression $a \oplus c \prec b \oplus c$ is equivalent to the inequality $(a \oplus c)-(b \oplus c)<0$, which can be simplified into:

$$
(1-c)(a-b)<0
$$

If $c \in[1,0]-\{\infty\}$ then $c \neq \infty$, but that means $c<1$, or $(1-c)(a-b)<0$ (because $a \prec b$ and $b \neq \infty)$. Thus, the above inequality is true, and we have proven $\mathbf{P}$.

Theorem 5. The algebra $\mathbf{B}=(\Lambda, \oplus)$ for Gilbert Channels and its total order $\preceq$ satisfy all the properties in $\mathbf{P}$, and thus compatible with the GDA.

PROOF: The proof is similar to that of theorem 4.
P1 We need to prove that if $\Lambda=[0,1] \times[0,1]$ then $\left(p_{1}, q_{1}\right) \oplus$ $\left(p_{2}, q_{2}\right) \in \Lambda$. First observe that $p\left(p_{1}, q_{1}, p_{2}, q_{2}\right)=p_{1} \oplus$ $p_{2}$. Recall that we have proven the closure property of this type of function in the previous theorem. Therefore, closure is satisfied iff $0 \leq q\left(p_{1}, q_{1}, p_{2}, q_{2}\right) \leq 1$. From (8)

$$
q=p \cdot \frac{X_{G}(e)}{1-X_{G}(e)} \leq 1
$$

Since $0 \leq X_{G}(e) \leq 1$, then $X_{G}(e) /\left(1-X_{G}(e)\right)>0$. In addition, if we assume that closure on $q$ is satisfied, then we have $0 \leq q \leq 1$, and the following must be true:

$$
\begin{aligned}
& q \leq 1 \\
& p \leq\left(1-X_{G}(e)\right) / X_{G}(e) \\
& p_{1}+p_{2}-p_{1} p_{2} \leq\left[\left(p_{1}+q_{1}\right)\left(p_{2}+q_{2}\right)-q_{1} q_{2}\right] /\left(q_{1} q_{2}\right) \\
& p_{1} q_{1} q_{2}+p_{2} q_{1} q_{2}-p_{1} p_{2} q_{1} q_{2} \leq p_{1} p_{2}+p_{1} q_{2}+p_{2} q_{1} \\
& p_{1} q_{1} q_{2}+p_{2} q_{1} q_{2} \leq p_{1} p_{2} q_{1} q_{2}+p_{1} p_{2}+p_{1} q_{2}+p_{2} q_{1} \\
& p_{1} q_{1} q_{2}+p_{2} q_{1} q_{2} \leq p_{1} q_{2}+p_{2} q_{1} \\
& q_{1}\left(p_{1} q_{2}\right)+q_{2}\left(p_{2} q_{1}\right) \leq p_{1} q_{2}+p_{2} q_{1}
\end{aligned}
$$

In the above, we first derived the second inequality using (11). Next, both sides of the inequality are expanded using (7). We then multiply both sides with $q_{1} q_{2} \geq 0$ and add $p_{1} p_{2} q_{1} q_{2}$ to both sides. At this point, we observe the inequality involves only positive terms. Removing two positive terms on the right hand side does not change the inequality. Finally, by appropriately factoring the terms on the left hand side, and using the fact that $q_{1}, q_{2} \leq 1$, we reach the final inequality that is always true.

To prove associativity, we can use a symbolic algebraic manipulator to verify that the following is true:

$$
\begin{gathered}
p\left(p\left(p_{1}, q_{1}, p_{2}, q_{2}\right), q\left(p_{1}, q_{1}, p_{2}, q_{2}\right), p_{3}, q_{3}\right)= \\
p\left(p_{1}, q_{1}, p\left(p_{2}, q_{2}, p_{3}, q_{3}\right), q\left(p_{2}, q_{2}, p_{3}, q_{3}\right)\right) \\
q\left(p\left(p_{1}, q_{1}, p_{2}, q_{2}\right), q\left(p_{1}, q_{1}, p_{2}, q_{2}\right), p_{3}, q_{3}\right)= \\
q\left(p_{1}, q_{1}, p\left(p_{2}, q_{2}, p_{3}, q_{3}\right), q\left(p_{2}, q_{2}, p_{3}, q_{3}\right)\right)
\end{gathered}
$$

Likewise, commutativity can be proven by verifying:

$$
\begin{aligned}
& p\left(p_{1}, q_{1}, p_{2}, q_{2}\right)=p\left(p_{2}, q_{2}, p_{1}, q_{1}\right) \quad \text { and } \\
& q\left(p_{1}, q_{1}, p_{2}, q_{2}\right)=q\left(p_{2}, q_{2}, p_{1}, q_{1}\right)
\end{aligned}
$$

and finally, it is easy to prove that for any $q \in[0,1]$, the element $(0, q)$ satisfies the property of the 0 element.

P2 The $\infty$ element is $(p, q)=(1,0) \in[0,1]$.

P3 Equation (8) defines $q$ as a linear function of $p$ with a slope of $X_{G}(e) /\left(1-X_{G}(e)\right)$. Thus, each value of $X_{G}(e)$ corresponds to a line $q(p)$ that intersects the origin $(p, q)=(0,0)$. These lines are therefore contour lines for the different values of $X_{G}(e) \in[0,1]$. The expression $\left(p_{1}, q_{1}\right) \preceq\left(p_{2}, q_{2}\right)$ then simply compares the slopes of the two lines (the steeper the slope, the better). P3 then follows from the ordering properties of $\mathbb{R}$.

P4 By P1, $(0, q)$ satisfies the criteria for the 0 element. If $e$ is an edge such that $\beta(e)=(0, q)$, then we have $X_{B}(e)<$ $X_{B}(f)$ for all edges $f$ with $\beta(f) \neq\left(0, q^{\prime}\right)$. 
P5 Suppose $a, b$, and $c$ are the values of $\beta\left(e_{1}\right), \beta\left(e_{2}\right)$, and $\beta\left(e_{3}\right)$, respectively. First, using the fact that $X_{B}=1-$ $X_{G}$, and recalling that $X_{G}(\cdot), X_{B}(\cdot) \in \mathbb{R}$, we prove the isotonicity property as follows:

$$
\begin{aligned}
a & \prec b \\
X_{B}(a) & \leq X_{B}(b) \\
X_{G}(a) & \geq X_{G}(b) \\
X_{G}(a) X_{G}(c) & \geq X_{G}(b) X_{G}(c) \\
X_{G}(a \oplus c) & \geq X_{G}(b \oplus c) \\
1-X_{G}(a \oplus c) & \leq 1-X_{G}(b \oplus c) \\
X_{B}(a \oplus c) & \leq X_{B}(b \oplus c) \\
a \oplus c & \prec b \oplus c
\end{aligned}
$$

Note that just as in our definition for $\oplus$, in our proof for isotonicity, we made heavy use of the relationship between the $\mathbf{B}$ and $\mathbf{X}$ algebras and their $\preceq$ operators.

Theorem 5 shows that the GDA can be used to reliably compute the network path that has the minimum long term channel erasure probability, which is an important QoS metric for a variety of modern communication systems. Mathematically, we state this result in the following corrolary:

Corrolary 6. The long term erasure probability $X(\boldsymbol{\lambda})$ is a non-decreasing function of its argument $\boldsymbol{\lambda}$. Consequently, the path with minimum $\boldsymbol{\lambda}$ obtained from the GDA is also the path with the minimum long term erasure probability $X(\boldsymbol{\lambda})$.

PROOF: The proof follows from our definition for $\preceq$ :

\section{CONCLUSION}

In this extended abstract, we have presented a method to find the best QoS path in a network where each link is modeled as a Gilbert channel. We briefly discussed why the Gilbert Channel Model is of considerable importance in modern communication systems. First, among the different models that are used to analyze a wide variety of communication channels with memory, the Gilbert Channel Model is one of the simplest and the most mathematically tractable. More importantly, the model was recently proposed as a tool for analyzing various QoS aspects of the multi-service UMTS-over-WCDMA networks.

Building from our previous body of work in the area of best-QoS routing for discrete memoriless channel networks, we proposed to use the Generalized Dijkstra's Algorithm to a class of communication network that can be modeled as a network of Gilbert Channels. This important class of network includes the TCP/IP networks and many types of wireless fading channel networks with correlated errors. In this extended abstract, we rigorously proved the algebraic correctness of our approach.

We described the various properties that are required in order to guarantee that the GDA can be used on any Gilbert Channel Networks. We proved that all compatibility properties are satisfied, and thus proved that the GDA returns the loop-free path with the best QoS in a network of Gilbert channels.
Future work includes extending the result to the general Finite-State Markov Chain channels and general Discrete Memoriless Channels. Numerical experiments can also be conducted to verify the correctness of this algebraic approach. In these experiments, our algorithm can be tested on random graphs with topologies that closely resemble the Internet. To generate such graphs, specialized random graph algorithms [16] can be used in combination with an edge weight generator that uses an experimentally determined distribution.

\section{ACKNOWLEDGMENTS}

This work is supported by the Caltech Lee Center for Advanced Networking and NSF Grant No. CCF-0514881. The author would like to thank Prof. Robert J. McEliece for useful discussions and his continued support for this research.

\section{REFERENCES}

[1] Gilbert, E. N., "Capacity of a burst-noise channel," Bell Syst. Tech. J., vol. 39, pp. 1253-1265, Sep. 1960.

[2] Swarts, J.S., Ferreira, H.C., "On the evaluation and application of Markov channel models in wireless communications," Vehicular Technology Conference, 1999. VTC 1999 - Fall. IEEE VTS 50th

[3] Tan, H.H., Hung, S.V., "Performance analysis of slotted ALOHA over fading communications channels," INFOCOM '90. Ninth Annual Joint Conference of the IEEE Computer and Communication Societies. Proceedings., IEEE

[4] Zorzi M., Rao R.R., "The Effect of Correlated Errors on the Performance of TCP" IEEE Communications Letters, Vol. 1, No.. 5, September 1997

[5] Lee K.K., Chanson S.T., "Packet loss probability for real-time wireless communications," IEEE Trans. Veh. Technol., vol. 51, no. 6, pp. 15691575, Nov. 2002.

[6] Turin, W. and Van Nobelen, R., "Hidden Markov modeling of fading channels," Proc. IEEE Veh. Technol. Conf., May 1998, pp. 1234-1238.

[7] ETSI, "Selection Procedures for the choice of radio transmission technologies of the UMTS," Universal Mobile Telecommunications System (UMTS); V3.2.0 (1998-04), Annex B.

[8] Gilhousen K.S., Jacobs I.M., Padovani R., Viterbi A.J., Weaver L.A.Jr., and Weatley C.E., "On the capacity of a cellular CDMA system," IEEE Trans. Veh. Technol., vol. 40, no. 2, pp. 303-312, May 1991.

[9] Evans J.S., Everitt D., "On the teletraffic capacity of CDMA cellular networks," IEEE T. Veh. Tech., vol. 48, no. 1, pp. 153-166, Jan. 1999.

[10] Elshabrawy, T., Le-Ngoc, T., "Capacity of future WCDMA networks supporting multimedia services," Selected Areas in Communications, IEEE Journal on, Volume 24, Issue 1, Jan. 2006 Page(s):54-64

[11] Elshabrawy, T., Le-Ngoc, T., "Gilbert channel approximation for downlink performance evaluation of WCDMA systems," Vehicular Technology Conference, 2005. VTC-2005-Fall. 2005 IEEE 62nd, Volume 1, 28-25 Sept., 2005 Page(s):382-386

[12] Sobrinho, J.L., "Algebra and algorithms for QoS path computation and hop-by-hop routing in the Internet," IEEE/ACM Transaction on Networking., vol. 10, pp. 541-550, August 2002.

[13] Cormen, T.H.; Leiserson, C.E.; Rivest, R.L., Introduction to Algorithms, MIT Press, MA and McGraw-Hill, NY, 1990.

[14] Soedarmadji, E., "Worst-Case Routing Performance Metrics for Sensor Networks," Pervasive Computing and Communications Workshops, 2007. PerCom Workshops '07. Fifth Annual IEEE International Conference on, 19-23 March 2007 Page(s):313-317

[15] Soedarmadji, E., "Optimal Routing in the Worst-Case-Error Metric", Global Telecommunications Conference, 2006. GLOBECOM '06. IEEE Nov. 2006 Page(s): $1-5$

[16] Jin C., Chen Q., Jamin S., "Inet: Internet Topology Generator," Tech Report UM-CSE-TR-433-00, University of Michigan, Ann Arbor 